\title{
Correction: Use of Deep Learning to Predict Acute Kidney Injury After Intravenous Contrast Media Administration: Prediction Model Development Study
}

Donghwan Yun ${ }^{1,2}$, MD; Semin $\mathrm{Cho}^{2}$, MD; Yong Chul Kim², MD, PhD; Dong Ki Kim², MD, PhD; Kook-Hwan $\mathrm{Oh}^{2}$, MD, PhD; Kwon Wook Joo ${ }^{2}$, MD, PhD; Yon Su Kim ${ }^{1,2}$, MD, PhD; Seung Seok Han ${ }^{1,2}$, MD, PhD

${ }^{1}$ Department of Biomedical Sciences, Seoul National University College of Medicine, Seoul, Republic of Korea

${ }^{2}$ Department of Internal Medicine, Seoul National University College of Medicine, Seoul, Republic of Korea

Corresponding Author:

Seung Seok Han, MD, PhD

Department of Biomedical Sciences

Seoul National University College of Medicine

103 Daehakro, Jongno-gu

Seoul, 03080

Republic of Korea

Phone: 82027408093

Email: hansway80@gmail.com

\section{Related Article:}

Correction of: https://medinform.jmir.org/2021/10/e27177

(JMIR Med Inform 2021;9(11):e34411) doi: 10.2196/34411

In "Use of Deep Learning to Predict Acute Kidney Injury After Intravenous Contrast Media Administration: Prediction Model Development Study" (JMIR Med Inform 2021;9(10):e27177), one error was noted.

In the originally published paper, names of 5 authors (Yong Chul Kim, Dong Ki Kim, Kwon Wook Joo, Yon Su Kim, and Seung Seok Han) were inadvertently formatted with middle initials instead of the full author names.

The full authorship list was listed as follows in the originally published paper.
Donghwan Yun, Semin Cho, Yong C Kim, Dong K Kim, Kook-Hwan Oh, Kwon W Joo, Yon S Kim, Seung S Han

This has been corrected to:

Donghwan Yun, Semin Cho, Yong Chul Kim, Dong Ki Kim, Kook-Hwan Oh, Kwon Wook Joo, Yon Su Kim, Seung Seok Han

The correction will appear in the online version of the paper on the JMIR Publications website on November 1, 2021, together with the publication of this correction notice. Because this was made after submission to PubMed, PubMed Central, and other full-text repositories, the corrected article has also been resubmitted to those repositories.

This is a non-peer-reviewed article. Submitted 21.10.21; accepted 26.10.21; published 02.11.21.

Please cite as:

Yun D, Cho S, Kim YC, Kim DK, Oh KH, Joo KW, Kim YS, Han SS

Correction: Use of Deep Learning to Predict Acute Kidney Injury After Intravenous Contrast Media Administration: Prediction Model Development Study

JMIR Med Inform 2021;9(11):e34411

URL: https://medinform.jmir.org/2021/11/e34411

doi: $10.2196 / 34411$

PMID: 
CDonghwan Yun, Semin Cho, Yong Chul Kim, Dong Ki Kim, Kook-Hwan Oh, Kwon Wook Joo, Yon Su Kim, Seung Seok Han. Originally published in JMIR Medical Informatics (https://medinform.jmir.org), 02.11.2021. This is an open-access article distributed under the terms of the Creative Commons Attribution License (https://creativecommons.org/licenses/by/4.0/), which permits unrestricted use, distribution, and reproduction in any medium, provided the original work, first published in JMIR Medical Informatics, is properly cited. The complete bibliographic information, a link to the original publication on https://medinform.jmir.org/, as well as this copyright and license information must be included. 